\title{
Efficacy of scroto-inguinal microsurgical varicocelectomy in treating male infertility
}

\author{
Hai Thanh Phan ${ }^{1}$, Thanh Xuan Nguyen ${ }^{1 *} \mathbb{0}$, Du Vinh Nguyen², Hoai Anh Vu', Duc Anh Le ${ }^{3}$ and Nhu Hien Pham ${ }^{1}$
}

\begin{abstract}
Background: Varicocele presents as the most frequent cause of infertility in men. Most reports showed that varicocelectomy has a significant impact on male fertility and reproductive outcome. This study aims to evaluate the safety and effectiveness of scrotal-inguinal microsurgical varicocelectomy in treating male infertility.

Methods: We prospectively studied preoperatively and postoperatively (at 3 and 6 months) 86 consecutive patients diagnosed with varicocele, abnormal semen parameters, and infertility, undergoing scrotal-inguinal microsurgical varicocelectomy. Semen test was performed before surgery and at 3 months and 6 months after surgery. The reproductive events were short-term followed up.

Results: The median age of the patient was 32.9 \pm 5.1 (20-43). Two cases (2.7\%) had a minor infection of the scrotum incision, who were well treated by appropriate antibiotics. After operation, total sperm count and the percentage of motile sperms at 3 months and 6 months were significantly higher than those pre-varicocelectomy, respectively. In total, $26.7 \%$ (23/86) of all couples achieved a spontaneous pregnancy. Late complications such as testicular atrophy, hydroceles, and recurrent varicocele have not occurred.
\end{abstract}

Conclusions: Scrotal-inguinal microsurgical varicocelectomy is an acceptable method in treating male infertility due to high rate of reproductive outcomes and very low rate of complications.

Keywords: Male infertility, Varicocele, Microsurgical varicocelectomy

\section{Background}

A varicocele is determined as abnormal tortuous and dilated veins in the pampiniform venous plexus of the scrotal sac. The prevalence of varicocele is between 15 and $20 \%$ of the men, up to $35 \%$ of male patients with infertility, and $75-81 \%$ of secondary male infertility [1]. It is proven that the presence of varicocele is correlated with a risen risk of abnormal sperm parameters [2-4]. Despite the mechanisms that have not yet been thoroughly described, it is possible to be multifactorial. Therefore, the management of varicoceles has been one of the resolutions of male reproductive medicine in the modern era.

\footnotetext{
*Correspondence: thanhxuanbvh@hotmail.com

${ }^{1}$ Department of Abdominal Emergency and Pediatric Surgery, Hue

Central Hospital, Hue City, Vietnam

Full list of author information is available at the end of the article
}

Varicoceles are now known as the most cause of male infertility that could be surgically repaired [5]. Several surgical techniques have been applied for varicocele treatment, such as open surgical ligation of the spermatic vein, microsurgical varicocelectomy, and laparoscopic varicocelectomy. Each method has its advantages and disadvantages, and different results have been reported in many previous studies [6-8].

Among varied surgical techniques, microsurgical varicocelectomy has been known as the golden standard for varicocele treatment recently because of its high rate of success and very low rate of complications $[6,9]$. The most common approaches are retroperitoneal abdominal laparoscopic, infra-inguinal, and sub-inguinal below the groin or intrascrotal [10, 11]. According to Fretz, the scrotal approach is no longer considered due to the higher risk of damage to the spermatic arteries and resultant testicular atrophy [12]. But from our experience, the 
scrotal-inguinal approach is easier, straightforward, and faster as compared to inguinal approach. Therefore, we carried out this study to evaluate the safety and effectiveness of scrotal-inguinal microsurgical varicocelectomy in treating male infertility.

\section{Methods}

\subsection{Study population}

Between January 2018 and February 2020, we prospectively studied preoperatively and postoperatively (at 3 and 6 months) 86 consecutive male patients with varicocele, abnormal semen parameters, and infertility, undergoing scrotal-inguinal microsurgical varicocelectomy. All operations were performed by only one urologist who had more than ten years of experience in microsurgical technique.

We included varicoceles according to the American Society for Reproductive Medicine and Society for Male Reproduction and Urology's Practice Committee report [4] when each of the following criteria are met: (1) The varicocele is palpable on physical examination of the scrotum; (2) the couple infertility for at least 12 months; (3) the male partner had at least one alteration semen parameter; (4) none of the other contributing male risk factors; and (5) his wife confirmed fertile at the date of evaluation. We excluded from this study the patients with azoospermia or with others like multiple risk factors, abnormality in his wife.

\subsection{Surgical technique}

The decision to treat was taken by an andrology team including andrologists, urologists, gynecologists, and biologists. The couple was provided with comprehensive information on the advantages and complications of the surgical technique. All patients underwent a unilateral microsurgical varicocelectomy.

Using either spinal or general anesthesia, two 2-cm incisions were made (scrotal and inguinal incision, as shown in Fig. 1). The spermatic cord was identified, and its fascia was opened. Under $10 \times$ microscopic

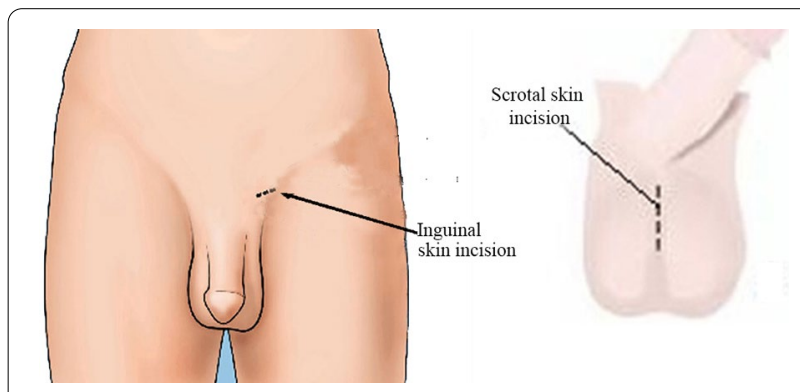

Fig. 1 Scrotal and inguinal skin incision magnification, the external and internal spermatic veins were identified (Fig. 2) and ligated using either sutures or surgical clips, while preserving the internal spermatic arteries, deferential artery, vasal vein, and lymphatic vessels (Fig. 3). Testicular biopsy was also performed in most cases to determine whether patients may benefit from varicocele repair $[13,14]$.

\subsection{Semen analysis}

A semen analysis was performed once before the surgery and at least two times after the surgery at the third and sixth month. Sperm count and total motile sperm count (TMSC) were calculated. The TMSC was calculated using the following formula: TMSC $=$ ejaculate volume $\times$ concentration $\times$ motile fraction.

Semen samples were examined within $60 \mathrm{~min}$ after ejaculation in order to maintain the quality of the specimen.

\subsection{Statistical analysis}

Outcome variables included: sperm concentration, percentage of motile sperms, spontaneous pregnancy, early and late complications. Postoperative complications were recorded and graded as per Clavien-Dindo classification. Subsequently, all patients were contacted by telephone in February 2020 to determine the reproductive events.

SPSS version 20 was used for statistical analysis. Descriptive statistics were used for semen health parameters at the 3- and 6-month stages. A paired $T$-test (or Wilcoxon test in case of not normally contributed data) was used to compare the evolution of sperm quality between the pre-operational level and the 3- and 6-month review.

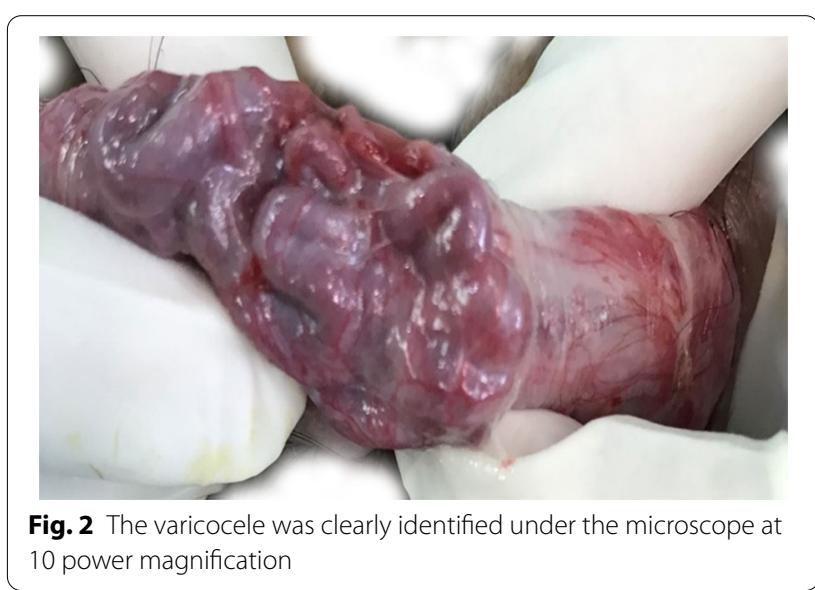



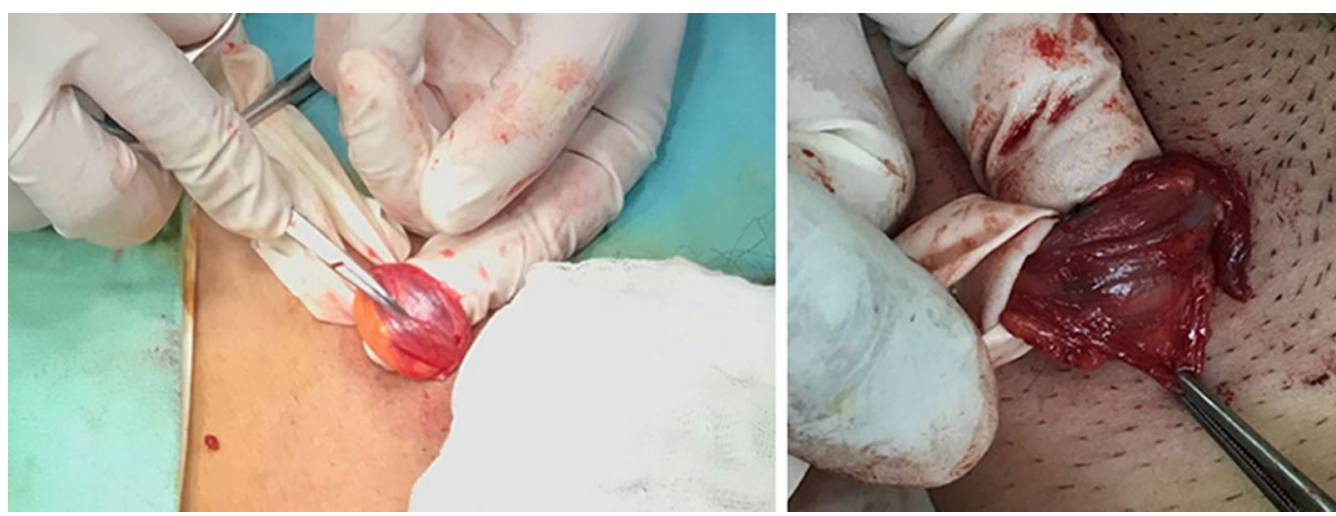

Fig. 3 The spermatic cord has been dissected through the inguinal skin incision

\section{Results}

A total of 86 consecutive men with palpable varicocele and infertility participated in our study. The median of age was $32.9 \pm 5.1$ years (range $20-43$ ). The pre- and postoperative semen parameters are shown in Table 1. After operation, the mean $( \pm S E)$ of total sperm count and the percentage of motile sperms at 3 months and 6 months were significantly higher than those pre-varicocelectomy.

The median follow-up period for reproductive outcome was 12.9 months (range 2-19 months).

The first spontaneous pregnancy in a couple was informed at 2 months after surgery. Cumulatively, 26.7\% $(23 / 86)$ of all couples achieved a pregnancy at the end of follow-up.

Out of the 63 patients that did not have pregnancy outcome data, 12 patients were histopathologically confirmed with testicular hyalinization. The remaining 51 patients were not significantly different from the 23 men with available pregnancy data with respect to initial sperm concentration, sperm motility, or age (male patients and spouse) (data not shown).

Postoperative events: Two cases $(2.7 \%)$ get a minor infection of the scrotum incision within one week after surgery, who were well treated with appropriate antibiotics. Late complications such as testicular atrophy, hydroceles, and recurrent varicocele have not occurred within a 6-month postoperative follow-up.

\section{Discussion}

The target of varicocele treatment is to obstruct the refluxing venous drainage to the testis while maintaining arterial inflow and lymphatic drainage as much as possible $[15,16]$. Many surgical techniques have been suggested. A number of studies have been reported, and they showed somewhat different outcomes, such as for pregnancy rates and potential complications [17]. According to Fretz, the scrotal approach is no longer considered to perform due to the higher risk of damage to the spermatic arteries and resultant testicular atrophy [12]. But in our experience, the scrotal-inguinal microsurgical varicocelectomy had many advantages that were proven in our series with a high success rate and very low potential complication. Furthermore, via the scrotal incision, the testicle was also examined in order to perform biopsy in most cases. This is the reason we prefer unilateral scrotal-inguinal microsurgical varicocelectomy in treating male infertility at our hospital recently to other surgical technique, such as inguinal microsurgical varicocelectomy. To our knowledge, this is the first paper using the scrotal-inguinal microsurgical technique for varicocelectomy.

The microsurgical varicocelectomy is an innovative technique that allows the ligation of all of the veins except the vasal vein while protecting the testicular artery and lymphatics, leading to a minimal rate of varicocele

Table 1 Preoperative and postoperative comparison of semen parameters

\begin{tabular}{|c|c|c|c|}
\hline Description & Preoperative values & 3 months & 6 months \\
\hline Number of patients & 86 & 62 & 41 \\
\hline Sperm count $\times 10^{6} / \mathrm{mL}$ (mean) & $10.01 \pm 4.8(0-178)$ & $21.19 \pm 6.8(0-160)$ & $16.96 \pm 5.3(0-110)$ \\
\hline ( $p$ value) & & $p=0.038$ & $p=0.001$ \\
\hline Motility\% (mean) & $3.88 \pm 1.2(0-28)$ & $12.52 \pm 2.3(0-37)$ & $16.04 \pm 2.9(0-40)$ \\
\hline ( $p$ value) & & $p=0.0001$ & $p=0.0001$ \\
\hline
\end{tabular}


recurrence and postoperative complications [18]. During microsurgical operation, delivery of the testicle provides direct visual access to all avenues of testicular venous effluent, which leads to a significantly decreased rate of varicocele recurrence [19].

Our data were consistent with most previous studies that varicocele repair had a positive impact on semen parameters. Agarwal et al. [20] conducted a meta-analysis from 17 studies to evaluate the efficacy of surgical varicocelectomy (inguinal microsurgery or high ligation) in developing semen parameters, including both observational studies and randomized clinical trials. The target population was men diagnosed infertile with clinically examined unilateral or bilateral varicocele and at least one alteration semen parameter. The results presented that the total sperm count increased by $9.71 \times 10^{6} /$ $\mathrm{mL}(p<0.00001)$, and sperm motility increased by 9.92\% $(p<0.0001)$ after performing a microsurgical varicocelectomy. Similarly, the sperm count increased by $12.03 \times 10^{6} / \mathrm{mL} \quad(p=0.0002)$, and sperm motility increased by $11.72 \%(p=0.002)$ after doing a high ligation varicocelectomy.

Baazeem et al. [21] carried out a similar meta-analysis study that consisted of only randomized clinical trials, but several studies for interventional radiology (embolization) as well as operation were included in their study. Sperm concentration, total sperm motility, and progressive motility before and after operation of clinical varicocele were investigated. The results suggested that varicocelectomy is correlated with a significant increase in sperm count, and total as well as progressive motility. In a meta-analysis conducted by Schauer et al. [22], they investigated the influence of 3 operative methods (sub-inguinal varicocelectomy, the inguinal approach, and high ligation) for varicocelectomy on sperm parameters (concentration and motility) and indicated that varicocelectomy could significantly improve the sperm concentration and motility regardless of operative methods. Besides, varicocele was correlated with sperm DNA damage, and this sperm pathology might be a secondary to varicocele-mediated oxidative stress. Some studies presented that varicocelectomy could reverse this sperm DNA damage [23].

Regarding the influence of varicocele treatment on the reproductive outcomes, our study showed $26.7 \%(23 / 86)$ of all couples achieved a spontaneous pregnancy within a short-term follow-up. The pregnancy rate in our study was lower than in other reports because out of the 63 patients that did not have pregnancy outcome data, 12 patients were histopathologically confirmed with testicular hyalinization. Therefore, diagnostic testicular biopsy during varicocele repair contributes a realistic and important role $[13,14]$.
In 2009, Cayan et al. [24] evaluated the pregnancy rate after varicocelectomy from 36 studies to find out the best surgical method based on the reproductive outcomes. They defined that the microsurgical varicocelectomy has a higher rate of spontaneous pregnancy and lower postoperative varicocele recurrence than conventional surgical methods and radiological intervention in treating male infertility.

In 2011, Diegidio et al. [25] analyzing from 33 studies with 5000 male patients with infertility showed similar results. The overall rate of pregnancy was reported to be $38.37 \%$, which was highest for the group performing microsurgical sub-inguinal and the microsurgical inguinal operation ( $44.75 \%$ and $41.78 \%$, respectively). Other surgical methods seem to obtain a lower rate of pregnancy outcomes compared with the microsurgical varicocelectomy. For example, the pregnancy rate with the laparoscopic technique was $27.5 \%, 30.1 \%$ for the conventional inguinal surgery, and $34.2 \%$ for the Palomo surgical technique.

Our study has some limitations. The small sample size, short-term follow-up, and lack of assisted reproductive techniques were known as the reasons for the spontaneous pregnancy rate in our study being lower than that in other reports. Furthermore, the absence of a control group made it less significant to prove efficacy of this surgical technique.

\section{Conclusion}

Our study highlights that scroto-inguinal microsurgical varicocelectomy is an excellent option for the treatment of male infertility because it has acceptable rates of reproductive outcomes and very low risk of complication. Further studies are strongly recommended in the field with a larger sample size and long-term follow-up to ascertain conclusions drawn from this study.

\section{Acknowledgements \\ The authors thank Dr. Nguyen Huu Son for his valuable assistance.}

\section{Authors' contributions}

HTP and TXN have contributed to format the design of the study and revised the collected data. DVN, HAV, DAL, and NHP have done the main work of the statistical data analysis. HTP, TXN, and NDV have shared the operational work of the study and writing works. HTP, TXN, and DVN have recruited the participants, contributed to the operational works, interpretation, and analysis of the results. All authors read and approved the final manuscript.

Funding

No funding resources

Availability of data and materials

The corresponding author has full access to all of the data and takes full responsibility for the veracity of the data.

\section{Ethics approval and consent to participate}

This study was approved by the Ethics Committee Review Board of Hue Central Hospital under the reference number of $01012018-\mathrm{HCH}$. Written consents 
were obtained from all patients. All couples were provided in detail the surgical procedure, success rate, costs, and likely complications.

\section{Consent for publication}

Not applicable.

\section{Competing interests}

The authors declare that they have no competing interests.

\section{Author details}

${ }^{1}$ Department of Abdominal Emergency and Pediatric Surgery, Hue Central Hospital, Hue City, Vietnam. ${ }^{2}$ Department of Assisted Reproduction - Andrology, Hue Central Hospital, Hue City, Vietnam. ${ }^{3}$ Department of General Surgery, Hue Central Hospital, Hue City, Vietnam.

Received: 9 June 2020 Accepted: 4 February 2021

Published online: 25 February 2021

\section{References}

1. Gorelick Jl, Goldstein M (1993) Loss of fertility in men with varicocele. Fertil Steril 59(3):613-616

2. Kantartzi PD, Goulis ChD, Goulis GD, Papadimas I (2007) Male infertility and varicocele: myths and reality. Hippokratia 11(3):99-104

3. Niederberger C (2005) Increased seminal reactive oxygen species levels in patients with varicoceles correlate with varicocele grade but not with testis size. J Urol 174(2):660

4. Practice Committee of the American Society for Reproductive M, Society for Male R, Urology (2014) Report on varicocele and infertility: a committee opinion. Fertil Steril 102(6):1556-1560

5. Schlesinger MH, Wilets IF, Nagler HM (1994) Treatment outcome after varicocelectomy. A critical analysis. Urol Clin North Am 21(3):517-529

6. Al-Kandari AM, Shabaan H, Ibrahim HM, Elshebiny YH, Shokeir AA (2007) Comparison of outcomes of different varicocelectomy techniques: open inguinal, laparoscopic, and subinguinal microscopic varicocelectomy: a randomized clinical trial. Urology 69(3):417-420

7. Cayan S, Kadioglu TC, Tefekli A, Kadioglu A, Tellaloglu S (2000) Comparison of results and complications of high ligation surgery and microsurgical high inguinal varicocelectomy in the treatment of varicocele. Urology 55(5):750-754

8. Unal D, Yeni E, Verit A, Karatas OF (2001) Clomiphene citrate versus varicocelectomy in treatment of subclinical varicocele: a prospective randomized study. Int J Urol 8(5):227-230

9. Wang J, Xia SJ, Liu ZH, Tao L, Ge JF, Xu CM, Qiu JX (2015) Inguinal and subinguinal micro-varicocelectomy, the optimal surgical management of varicocele: a meta-analysis. Asian J Androl 17(1):74-80
10. Velasquez M, Tanrikut C (2014) Surgical management of male infertility: an update. Transl Androl Urol 3(1):64-76

11. Leslie SW, Sajjad H, Siref LE (2020) Varicocele. In: StatPearls. Treasure Island.

12. Fretz PC, Sandlow II (2002) Varicocele: current concepts in pathophysiology, diagnosis, and treatment. Urol Clin North Am 29(4):921-937

13. Hwang K, Lamb DJ (2011) Diagnostic testicular biopsy before varicocele repair plays a realistic and important role. Fertil Steril 95(2):488

14 Inci K, Gunay LM (2013) The role of varicocele treatment in the management of non-obstructive azoospermia. Clinics (Sao Paulo) 68(Suppl 1):89-98

15. Masson P, Brannigan RE (2014) The varicocele. Urol Clin North Am 41(1):129-144

16. Evers JL, Collins JA (2003) Assessment of efficacy of varicocele repair for male subfertility: a systematic review. Lancet 361 (9372):1849-1852

17. Cho KS, Seo JT (2014) Effect of varicocelectomy on male infertility. Korean J Urol 55(11):703-709

18. Choi WS, Kim SW (2013) Current issues in varicocele management: a review. World J Mens Health 31(1):12-20

19. Beck EM, Schlegel PN, Goldstein M (1992) Intraoperative varicocele anatomy: a macroscopic and microscopic study. J Urol 148(4):1190-1194

20. Agarwal A, Deepinder F, Cocuzza M, Agarwal R, Short RA, Sabanegh E, Marmar JL (2007) Efficacy of varicocelectomy in improving semen parameters: new meta-analytical approach. Urology 70(3):532-538

21. Baazeem A, Belzile E, Ciampi A, Dohle G, Jarvi K, Salonia A, Weidner W, Zini A (2011) Varicocele and male factor infertility treatment: a new metaanalysis and review of the role of varicocele repair. Eur Urol 60(4):796-808

22. Schauer I, Madersbacher S, Jost R, Hubner WA, Imhof M (2012) The impact of varicocelectomy on sperm parameters: a meta-analysis. J Urol 187(5):1540-1547

23. Zini A, Dohle G (2011) Are varicoceles associated with increased deoxyribonucleic acid fragmentation? Fertil Steril 96(6):1283-1287

24. Cayan S, Shavakhabov S, Kadioglu A (2009) Treatment of palpable varicocele in infertile men: a meta-analysis to define the best technique. J Androl 30(1):33-40

25. Diegidio P, Jhaveri JK, Ghannam S, Pinkhasov R, Shabsigh R, Fisch H (2011) Review of current varicocelectomy techniques and their outcomes. BJU Int 108(7):1157-1172

\section{Publisher's Note}

Springer Nature remains neutral with regard to jurisdictional claims in published maps and institutional affiliations.

\section{Submit your manuscript to a SpringerOpen ${ }^{\circ}$ journal and benefit from:}

- Convenient online submission

- Rigorous peer review

- Open access: articles freely available online

- High visibility within the field

- Retaining the copyright to your article

Submit your next manuscript at $\boldsymbol{\nabla}$ springeropen.com 